\title{
YouTube e influencers en la infancia. Análisis de contenidos y propuestas educativas
}

YouTube and influencers in childhood. Content analysis and educational proposals

\section{YouTube e influenciadores na infância. Análise de conteúdo e propostas educacionais}

\author{
Dra. Paula Renés Arellano \\ Profesora Contratada Doctora \\ (Universidad de Cantabria) \\ http://orcid.org/0000-0003-0932-7694 \\ España \\ Dr. Vicent Gozálvez Pérez \\ Profesor del departamento de Teoría de la Educación \\ (Universidad de Valencia) \\ https://orcid.org/0000-0002-7952-8347 \\ España \\ Dra. Inmaculada Berlanga Fernández \\ Profesora Titular \\ (Universidad Internacional De La Rioja) \\ https://orcid.org/0000-0002-0135-624X \\ España
}

Fecha de recepción: 8 de octubre de 2019

Fecha de revisión: 26 de enero de 2020

Fecha de aceptación: 28 de marzo de 2020

Fecha de publicación: 1 de julio de 2020

Para citar este artículo: Renés Arellano, P., Gozálvez Pérez, V. y Berlanga Fernández, I.

(2020). YouTube e influencers en la infancia. Análisis de contenidos y propuestas educativas, Icono 14, 18 (2), 269-295. doi: 10.7195/ri14.v18i2.1455 


\section{Resumen}

La presencia de las tecnologías móviles e Internet entre los niños y jóvenes está generando nuevos marcos de comprensión, interacción y consumo de medios y contenidos. YouTube se ha convertido en una de las plataformas más utilizadas por estos, lo cual exige investigar las repercusiones ejercidas por estos influencers en los niños. En el presente trabajo se realiza una contextualización teórica sobre el nuevo entorno digital en el que Internet y las redes sociales forman parte indiscutible de la cotidianeidad, para avanzar hacia un marco metodológico y de investigación de los principales YouTubers seguidos por niños/as de Educación Primaria en España. Se ha utilizado una metodología cuantitativa, para conocer los principales YouTubers, y cualitativa, para analizar los perfiles de dichos influencers. Los resultados indican la predilección por determinados YouTubers españoles y el empleo, por parte de la mayoría, de videojuegos o bromas asociados a sus canales. Finalmente, se ofrece una reflexión sobre el contenido consumido por niños en Internet a través de estos canales, aportando propuestas pedagógicas para favorecer una educación mediática adecuada para el desarrollo sociomoral en la infancia.

Palabras clave: Redes sociales; Educación Primaria; Educación mediática; Desarrollo moral; Dispositivos móviles; Escuela

\section{Abstract}

The presence of mobile technologies and Internet among children and young people is generating new frames of understanding, interaction and consumption of media and content. YouTube has become one of the platforms most used by these, which requires investigating the repercussions exerted by these influencers on children. In the present work, an analysis of the main YouTubers followed by children of Primary Education in Spain is carried out. It has used a quantitative methodology to know the main YouTubers, and qualitative, to analyze the profiles of these influencers. The results indicate the predilection Spanish YouTubers and that most use videogames or jokes associated with their channels. Finally, a reflection is offered on the content consumed by children on the Internet through these channels, providing pedagogical proposals to develop an adequate media education for psychosocial child development.

Key Words: Social networks; Primary Education; Media education; Moral development; Mobile learning; School 
YouTube e influencers en la infancia. Análisis de contenidos y propuestas educativas | 271

MONOGRÁFICO

\section{Resumo}

A presença de tecnologias móveis e da Internet entre crianças e jovens está gerando novas estruturas para compreensão, interação e consumo de mídia e conteúdo. 00 YouTube se tornou uma das plataformas mais usadas por elas, o que exige investigar as repercussões exercidas por esses influenciadores nas crianças. Neste artigo, é realizada uma contextualização teórica sobre o novo ambiente digital no qual a Internet $e$ as redes sociais são uma parte inquestionável da vida cotidiana, para avançar em direção a uma estrutura metodológica e de pesquisa dos principais YouTubers seguidos por crianças do ensino fundamental na Espanha. Foi utilizada uma metodologia quantitativa, para conhecer os principais YouTubers e qualitativa, para analisar os perfis desses influenciadores. Os resultados indicam a preferência por determinados YouTubers em espanhol e o uso, pela maioria, de videogames ou piadas associadas a seus canais. Por fim, é oferecida uma reflexão sobre o conteúdo consumido pelas crianças na Internet por meio desses canais, apresentando propostas pedagógicas para favorecer uma educação midiática adequada ao desenvolvimento sociomoral na infância.

Palavras chave: Redes sociais; Educação primária; Educação para a mídia; Desenvolvimento moral; Dispositivos móveis; Escola

\section{Introducción: La explosión global en comunicación móvil, las redes sociales y las plataformas online (YouTube)}

El nuevo entorno digital caracterizado por la omnipresente conexión a Internet ha supuesto una explosión de modos de socialización que se suman a los tradicionales, especialmente con la irrupción de las redes sociales a través de los dispositivos móviles (tablets y smartphones). En este artículo se intenta primeramente explorar las nuevas formas de socialización en niños de Primaria (de edades comprendidas entre los 9 y los 12 años) unidas a las redes sociales, especialmente YouTube, con el fin de adentrarnos en los rasgos de tal socialización, sobre todo en un sentido práctico y ético (hábitos de uso, formas de relación, mensajes e imágenes que se transmiten, y valores que subyacen a las mismas). A partir de ahí y en segundo lugar, el propósito que se persigue va más allá de la descripción sociológica o fáctica del proceso de nueva socialización, y apunta (1) al análisis de la dimensión educativa de las redes sociales (eminentemente 
YouTube), en tanto que las redes sociales educan informalmente, y (2) a las orientaciones operativas, válidas o de interés para los agentes con responsabilidad educativa (familias, profesores, instituciones o centros que trabajan con menores, etc.).

Los nuevos dispositivos móviles con conexión a Internet han transformado nuestro día a día, pero ¿en qué términos está ocurriendo esta transformación? ¿Cuáles son las oportunidades y retos que plantea? ¿Cuáles sus peligros y sus beneficios? Dado el dinamismo de los cambios en torno a la nueva realidad tecnocomunicativa, estas preguntas tendrán que ser replanteadas constantemente, reconsiderando los criterios que auxilian en la evaluación de tal realidad.

La tecnología móvil con conexión a Internet ha introducido nuevos hábitos en la vida de la ciudadanía. El Smartphone reúne de modo convergente múltiples formas de comunicación como la transmisión de imágenes y vídeos, la navegación por la Red, además de la conexión telefónica, tendencia que en 2012 fue anunciada por numerosos investigadores (Luengo de la Torre, 2012). El proceso de convergencia digital ha modificado el espectro en cuanto a formas de comunicación y, la difusión y arraigo de la tecnología móvil a nivel mundial es un fenómeno que muy previsiblemente va a consolidarse en los próximos años, tal como establece el informe Horizon-k-12 (Johnson, Adams, Estrada \& Freeman, 2014).

Según la ITU-2018 (International Telecommunication Union), agencia de Naciones Unidas especializada en las tecnologías de la información y la comunicación, el porcentaje de suscripciones a dispositivos móviles ha crecido exponencialmente a nivel global: por ejemplo, en países tecnológicamente avanzados como son los asiáticos, el número de suscripciones por 100 habitantes pasó de 22,6 en el año 2005 a 109,7 en 2018. Un aumento parecido se observa en los Estados Árabes de 26,8 en 2005 a 103,1 en 2018 y en Europa este porcentaje también ha sido alto de 89,3 en 2005 a 120 en 2018.

De acuerdo con el Informe Ditrendia: Mobile en España y en el Mundo (2018), el número de dispositivos móviles a nivel global alcanzó los 5.135 mil millones, lo que significa que el $68 \%$ de la población mundial ya cuenta con un móvil. Y si en Europa 78 de cada 100 habitantes cuenta con un teléfono Smartphone, en España los teléfonos móviles inteligentes representan ya el $97 \%$ del total de teléfonos móvi- 
YouTube e influencers en la infancia. Análisis de contenidos y propuestas educativas | 273 MONOGRÁFICO

les, lo que sitúa a nuestro país en el sexto lugar del ranking, encabezado por Corea del Sur, Hong Kong, Italia, Singapur, Polonia y Tailandia, y entre los tres primeros a nivel europeo. Lo cierto es que más de 29 millones de personas en España tienen un Smartphone. Atendiendo a los últimos datos de 2019 del portal de estadísticas Statista, la cifra asciende ya a los 2.659,4 millones de usuarios poseen teléfonos inteligentes. Igualmente, el aumento de tabletas digitales en nuestro país ha sido considerable, a pesar de que hay un ligero retroceso en las ventas (un 7,9\%): tres de cuatro hogares disponen de Tablet.

En el gráfico siguiente (ITU-2019), podemos observar de modo muy ilustrativo el aumento en suscripciones de telefonía móvil a nivel global en los últimos años (una media de 108 suscripciones por 100 habitantes en 2019), sin duda superior al experimentado en otros ámbitos como las suscripciones de telefonía fija:

Evolution of mobile and fixed subscriptions, 2005-2019

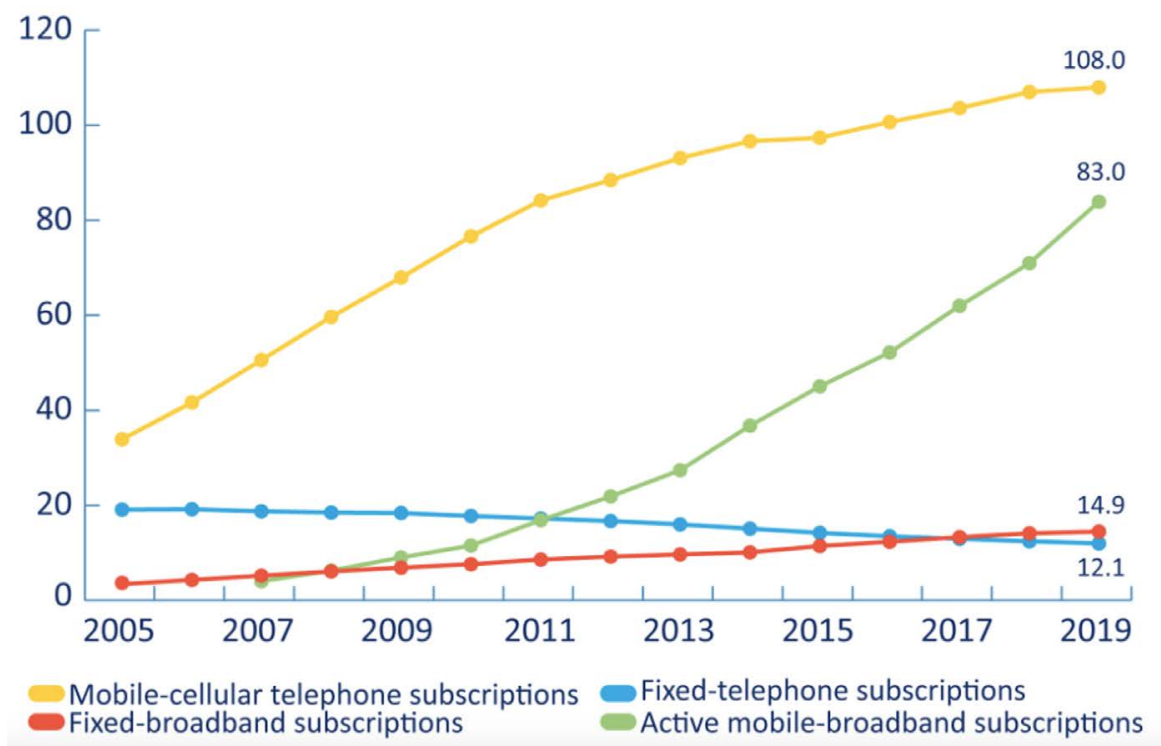

Figura 1: Global ICT developments 2005-2019. Fuente: ITU World Telecommunication (2019). 
De igual modo crece el número de conexiones a Internet. El uso de las redes sociales en nuestro país se ha disparado en los últimos años, de modo que los internautas españoles se conectan cada semana a una media de 6,2 plataformas sociales, cifra superior a la media europea $(5,4)$ y a la media mundial $(4,8)$, tal como se desprende del informe Connected Life, elaborado por Kantar TNS y difundido por la web de RTVE. Según este mismo informe, basado en 70.000 entrevistas pasadas a internautas de 57 países, las redes más usadas son WhatsApp (un $86 \%$ de internautas la usa cada semana vía smartphone), Facebook (83\%), YouTube (72 $\%$ ), Twitter (45\%) y Facebook Messenger (43\%). De los internautas españoles encuestados, un $97 \%$ disponía en 2016 de teléfono inteligente y un $61 \%$ de tableta. Pero los datos tienen alcance más amplio, no sólo reducido a países tecnológica o económicamente avanzados, tal como se desprendía del informe de la ITU. De acuerdo con Connected Life, a nivel global el $85 \%$ de la población internauta tiene un Smartphone, cifra superior en siete puntos a la registrada en 2015, es decir, un año antes. Y en 2019 publica que el $37 \%$ de los jóvenes afirma que usa demasiado el móvil. De este modo, la revolución comunicativa a la que estamos asistiendo no puede por menos que ayudar a reconfigurar nuestro mundo: si aceptamos con Habermas (2009) y su teoría de la acción comunicativa que el ser humano viene definido por su dimensión simbólica y comunicativa en un entorno cultural dado, todo apunta a que los perfiles comunicativos de la nueva cultura digital configurarán en buena parte la reproducción o transformación cultural, la interacción social y la formación de la personalidad: es decir, la manera de ser, relacionarse y entenderse a sí mismos los agentes del nuevo entorno comunicacional. Lo cual no deja de tener consecuencias en la construcción de la personalidad moral de la infancia y juventud, siguiendo parámetros cognitivos clásicos y más actuales (Turiel, 1998; Kurtines \& Gewirtz, 1991; Kohlberg, 1984; Harrison, 2014; Morgan \& Kristjánsson, 2017; Arthur, Kristjánsson, Harrison, Sanderse \& Wright, 2017). A ello nos dedicaremos en el apartado que sigue.

\section{Redes sociales, desarrollo sociomoral e infancia: el caso YouTube}

Una vez constatado el fenómeno de la irrupción social de la tecnología móvil, de indudables dimensiones globales, lo que interesa ahora es continuar con las investigaciones 
YouTube e influencers en la infancia. Análisis de contenidos y propuestas educativas | 275 MONOGRÁFICO

acerca de las consecuencias a nivel social, moral y educativo de tal fenómeno, especialmente en referencia al uso ético de las redes sociales a través de dispositivos móviles con conexión a Internet. El estudio presentado se centra en la red social YouTube y su uso en la infancia media (de los 6 a los 12 años), colectivo vulnerable que, aun conociendo en ocasiones los riesgos del uso inadecuado de Internet, navega por la Red al margen de los peligros que puede acarrear (Sabater, 2014; De-Frutos \& Marcos, 2017).

YouTube es una red social con perfil propio, pues en ella lo que determina el vínculo entre usuarios no es el texto escrito, sino el relato audiovisual (vídeos) en el que los jóvenes difunden ideas, creencias y costumbres (Vizcaíno-Verdú, Contreras \& Guzmán-Franco, 2019). Desde su creación en febrero de 2005, con un primer vídeo de 18 segundos que mostraba al cofundador Jawed Karim en el zoológico de San Diego, YouTube se ha convertido un espacio en el que los usuarios cuelgan y comparten vídeos (unas 300 horas de vídeo cada minuto en todo el mundo) y que plantea otro formato y otra narrativa, más allá de la tradicional comunicación lectoescritora. Se comparten vídeos de contenido cultural, lúdico, informativo, formativo, demostrativo o instructivo... en una variedad inabarcable de temas y contenidos. A nivel mundial, se baraja la cifra de 1000 millones de usuarios de YouTube, un tercio del total de internautas $\mathrm{y}$, lo interesante para nuestro estudio, más de la mitad de las visitas se realizan desde un Smartphone (según el portal informativo de YouTube: youtube.com). Tal como anunciaba el Informe ditrendia: Mobile en España y en el Mundo (2016), las perspectivas ese año apuntaban a que para 2019 el vídeo móvil supondría el $72 \%$ de todo el tráfico global de datos móviles, por lo que es de esperar que la red social YouTube jugará en este sentido un papel predominante.

En 2017 los datos publicados por entidades que miden el tráfico y la popularidad de páginas en Internet de todo el mundo indican que tanto en el informe de ComScore, "Jerarquía de Necesidades en Móvil" así como los datos que maneja Alexa.com a nivel internacional en tiempo real, YouTube es una de las cinco plataformas que lideran el ranking mundial de consumo por parte de las personas. El análisis de consumo de YouTube en Reino Unido indica que dicha plataforma es la primera entre los usuarios mayores de 18 años en la visualización de vídeos en todos los segmentos demográficos (Morgan \& Kristjánsson, 2017). Los estudios sobre el consumo de YouTube entre menores y su repercusión en su desarrollo sociomoral son escasas (Blackwell et al., 2014). El 
desarrollo sociomoral se define como la evolución y rasgos de la comprensión y valoración de eventos sociales con repercusiones morales, es decir, valoraciones referidas al daño propio y de los otros, a los vínculos interpersonales y a las relaciones de inclusión o exclusión, así como al seguimiento de los estándares sociales de justicia o corrección en la conducta (Turiel, 1998; Nucci, 2001; Yáñez-Canal et al., 2013).

Desde la investigación pedagógica acerca de las redes sociales en la infancia, quisiéramos mencionar el trabajo de Blackwell et al. (2014), quienes demostraron que YouTube y Facebook eran las redes sociales más usadas por niños de entre 8 y 12 años, aunque con diferentes usos y con diferentes contenidos según edad y sexo, de acuerdo con los intereses y desarrollo emocional, social y cognitivo de los menores. El segmento de edad comprendido entre 10 y 12 años visitaba las redes sociales con más frecuencia al día ( 3 o más horas) que los más pequeños ( 8 a 10 años), que visitaban las redes un promedio de 1-2 horas al día. El tipo de contenidos según franja de edad se correspondía con el desarrollo sociomoral de los niños, de modo que los más mayores (10 a 12 años) empleaban más tiempo en sitios de videoclips y películas y en sitios de redes sociales. En cambio, los más pequeños (8-10 años) visitan con mayor frecuencia sitios web de televisión, virtuales, de juguetes, y sitios Web educativos. Mientras que los niños de 11 a 12 años buscan a través de la red mayor independencia, más interacción con los pares y mayor experimentación con la identidad, los niños de 8 a 10 años todavía tienden a actividades infantiles centradas en la televisión infantil y el juego imaginario.

En la misma línea, se ha investigado el uso de YouTube en relación con el desarrollo sociomoral en la infancia. Resultan interesantes las investigaciones de Koh (2014), quien, tras estudiar las respuestas de los niños ante fragmentos de videos buscados y extraídos de YouTube, llegó a la conclusión de que los sitios web para compartir vídeos (YouTube) ofrecen oportunidades para la percepción, valoración y discusión de cuestiones morales relevantes, compartidas y comentadas a nivel global. Observó que los comentarios ante vídeos compartidos reflejaban el nivel de juicio y razonamiento moral, así como el desarrollo de la identidad personal (imagen de sí mismo). Y aquí la conclusión más interesante: “Desde un punto de vista educativo, YouTube allana el camino para usar materiales de vídeo apropiados para promover valores morales y el desarrollo moral" (Koh, 2014: 619), de acuerdo con el paradigma 
YouTube e influencers en la infancia. Análisis de contenidos y propuestas educativas | 277 MONOGRÁFICO

cognitivo de Piaget y Kohlberg (Kohlberg, 1984; Turiel, 1998) por un lado, y con las propuestas del desarrollo de la identidad personal de Loevinger (1987) por otro.

Poco antes, Banaji (2013) analizó el potencial educativo de YouTube en la dimensión sociomoral y cívica. Esta red social para compartir vídeos tiene la virtualidad de otorgar una dimensión social y pública a situaciones concretas, grabadas y subidas a la Red, con una carga moral indiscutible: situaciones que generan angustia, perplejidad e indignación pues contienen secuencias que muestran conflictos interpersonales, actitudes racistas o discriminatorias, y que despiertan la discusión moral acerca de los prejuicios, de la clase social, del nacionalismo o la inmigración. Motivo por el cual Banaji (2013: 69) concluye que una red social como YouTube supone un "recurso significativo, aunque todavía no considerado, en la educación para la ciudadanía, tanto la formal como la informal, debido a sus compromisos con la tecnología, el contexto social, el contexto emocional y la retórica política". Por ejemplo, Zahn et al. (2014) demostraron que la creación y el uso colaborativos de videoclips a través de YouTube ofrecían inmejorables oportunidades educativas para la adquisición de conocimiento y el cambio de actitud en torno a un problema de salud pública como la obesidad. Sin embargo, y desde una perspectiva centrada sólo en habilidades cognitivas, parece ser que el uso habitual de YouTube en la infancia no contribuye tanto como Facebook a la estimulación de habilidades comunicativas como la capacidad verbal, la memoria operativa (working memory) y la ortografía (Alloway, Horton, Alloway \& Dawson, 2013).

Por otra parte, y en relación las redes sociales en general, desde el Jubilee Center for Character and Virtues, de la Universidad de Birmingham, se realizó en 2016 una encuesta en Reino Unido que evidenció que la mayoría de los padres consideran que el acceso a las redes sociales por parte de sus hijos tiene un efecto cuestionable en su desarrollo moral y afectivo. Previamente, se realizó otro estudio con chicos de entre 11 y 14 años, los cuales afirmaron que las reglas y recomendaciones acerca del buen uso de las redes sociales son opacas o confusas. Además, se evidenció que los chicos de estas edades no son conscientes de las consecuencias de algunas de sus acciones en la red, por ejemplo, del daño que pueden ocasionar los mensajes que envían o suben a las redes sociales (Harrison, 2014; Arthur, Kristjánsson, Harrison, Sanderse \& Wright, 2017; Morgan \& Kritjánsson, 2017; Harrison, 2016). 
Ante estas cifras, y tomando en consideración que los niños y jóvenes se conectan a las redes sociales a través de dispositivos móviles (Holloway, Green \& Livingstone, 2013; Briggs, 2017; Santana-Vega, Gómez-Muñoz \& Feliciano-García, 2019), no es de extrañar que el Smartphone despierte el interés de las ciencias sociales, y su auge, ventajas o riesgos se conviertan en objeto de estudio de las más diversas disciplinas, conocedoras del papel relevante que juegan las tecnologías emergentes a la hora de educar o formar a la sociedad en ciertos valores, especialmente los valores éticos y cívicos, fundamentales en democracia (Gozálvez \& Jover, 2016). Este es el motivo por el que en el presente estudio tratamos de analizar los contenidos y valores que los influencers vuelcan en la red social YouTube, especialmente a través de videos seguidos por menores de Educación Primaria (9 a 12 años) en España, con el fin de proponer acciones educativas que favorezcan el desarrollo sociomoral y cívico en menores conectados vía Smartphone a YouTube.

\section{Material y Método}

Analizado este fenómeno, surgen cuestiones vinculadas a lo que realmente está sucediendo cuando un niño consume o crea materiales audiovisuales o mediáticos en estas redes y de cómo influyenen los comportamientos de niños y niñas. El objetivo es analizar qué contenidos son los que con más frecuencia consumen los menores al estar expuestos a ciertos YouTubers. El enfoque metodológico empleado es cualitativo, siguiendo las aportaciones del modelo de análisis de la comunicación de la red social de Pérez-Escoda y Contreras (2018) sobre Musical.ly y Katz y Lazarfel en Rodrigo (2001) sobre la influencia que ejercen algunos influencers en sus seguidores. En este caso de estudio, la plataforma elegida por sus potencialidades ha sido YouTube, para lo cual se han analizado las características de los perfiles más populares entre los elegidos por niños entre 9 y 12 años que han participado en el estudio para finalizar ofreciendo resultados y propuestas educativas que permitan hacer un uso adecuado de Youtube.

\section{Muestra}

El proceso y desarrollo de la investigación se centró en diseñar y elaborar un cuestionario para recoger los datos vinculados a YouTube por parte de 140 niños de Educación Primaria entre 9 y 12 años seleccionados de forma aleatoria en diferentes comunidades autónomas de España. A través de los resultados obtenidos se 
YouTube e influencers en la infancia. Análisis de contenidos y propuestas educativas | 279 MONOGRÁFICO

detectaron los perfiles YouTubers más seguidos por estos y se procedió a realizar el análisis de contenido por parte de un panel de expertos.

\section{Instrumentos}

Tomando como referencia el método de análisis documental descriptivo de López Noguero (2012), se usaron criterios de rigurosidad y sistematicidad elaborando una rúbrica para evaluar los perfiles de los YouTubers seleccionados por los niños encuestados, realizando una validación del contenido con expertos que fueron seleccionados mediante muestreo intencional. Empleando el criterio de inclusión se seleccionaron cinco profesionales universitarios expertos del ámbito educativo, audiovisual y estadístico, con más de ocho años de experiencia profesional y con conocimientos sobre los instrumentos objeto de validación a través de un panel Delphi online como técnica de consenso. Las variables de estudio fueron: YouTubers más seguidos por los niños de entre 9 y 12 años, año de nacimiento, otras redes sociales disponibles, suscriptores, fecha de creación y vídeos creados y compartidos, destinatarios, presencia y representación de empresas en sus canales, sexo, identificación de perfiles (fotografías, avatares, imágenes...), tipos de vídeos compartidos, análisis de contenidos de vídeos, opciones de interfaz para comunicarse con sus seguidores y valores transmitidos.

\section{Procedimiento}

Se ha usado una metodología hermenéutica (Conill, 2006) con el fin de interpretar los comentarios e interacciones mediáticas de los influencers, vinculando de este modo la descripción empírica con la interpretación y evaluación de acuerdo con las pautas del desarrollo sociomoral -pautas que se corresponden con los criterios de una ética cívica basada en el respeto activo, en las libertades personales y en el reconocimiento del otro como igual y digno (Zayas, Gozálvez \& Gracia, 2019).

De modo complementario, los criterios para evaluar este desarrollo los extraemos de las propuestas de la tradición cognitiva pero actualizada de acuerdo con parámetros más amplios, desde la ética del cuidado, el enfoque de las capacidades, la ética dialógica y la perspectiva intercultural (Gozálvez \& Jover, 2016; Nussbaum, 2012; Cortina, 2007 y 2010; Benhabib, 2005). 


\section{Resultados}

Tomando como referencia los resultados obtenidos de los cuestionarios respondidos por los niños y niñas de entre 9 y 12 años sobre qué YouTubers suelen seguir, las respuestas se concentran principalmente en los influencers de origen español tales como Vegetta777 o elrubiusOMG, como se recoge en la Figura II. En concreto, elrubiusOMG y AuronPlay son los YouTubers en los que se coindice como los más seguidos en España por los niños y adolescentes, coincidiendo de esta manera con los resultados de la muestra obtenida. Una característica de estos seis YouTubers con alto seguimiento (influencers) es que todos tienen edades parecidas, nacidos en torno a los años 90, y la mayoría disponen de otras redes además de YouTube como es Facebook, Instagram, Twitter o Google Plus, siendo Wismichu y DjMariio quienes además poseen cuenta de TV en Twitch, siendo este último propietario de un segundo canal de YouTube llamado DjMaRiioOHD.

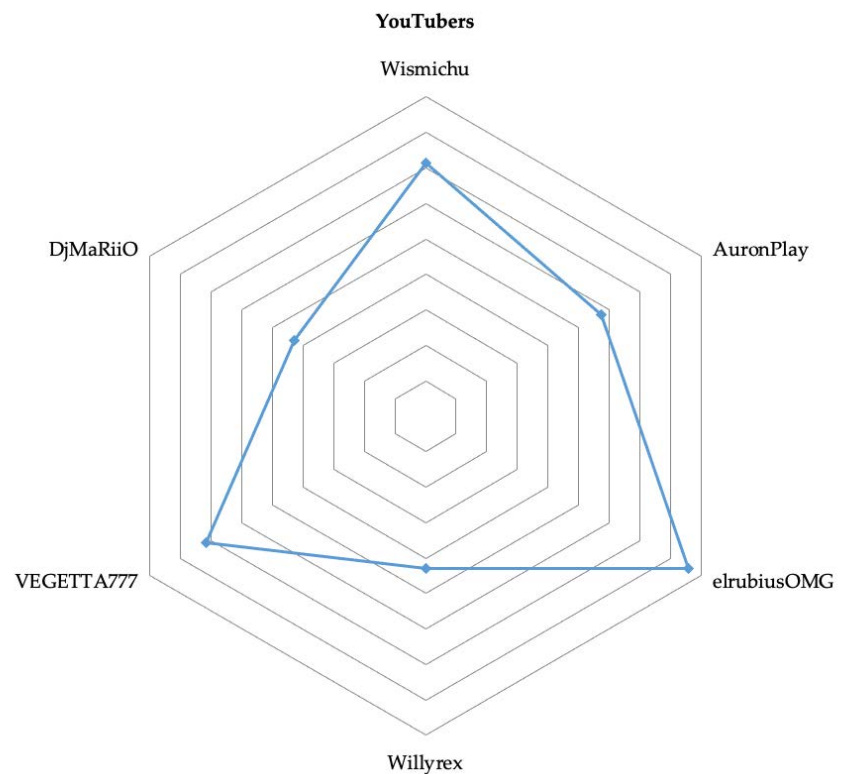

Figura 2: YouTubers seguidos por niños y niñas de Educación Primaria.

Fuente: Elaboración propia.

A partir de la detección de los YouTubers más seguidos por los niños/as que han respondido al cuestionario, se ha procedido a realizar una tabla con los datos más 
relevantes de estos en cuanto a suscriptores, fecha de creación y vídeos creados y compartidos en los canales propios de cada uno de ellos (véase Tabla I).

\begin{tabular}{|c|c|c|c|c|c|}
\hline YouTubers & País & Suscriptores & $\begin{array}{l}\text { Fecha } \\
\text { creación }\end{array}$ & $\begin{array}{c}\mathrm{N}^{\circ} \\
\text { Vídeos }\end{array}$ & Canal \\
\hline Wismichu & España & $6,551,843$ & $22 / 01 / 2012$ & 281 & $\begin{array}{c}\text { https://www.youtube.com/ } \\
\text { user/wismichu/ }\end{array}$ \\
\hline AuronPlay & España & $6,807,853$ & $28 / 02 / 2006$ & 299 & $\frac{\text { https://www.youtube.com/ }}{\text { user/AuronPlay/ }}$ \\
\hline elrubius0MG & España & $26,979,227$ & $19 / 12 / 2011$ & 751 & $\frac{\text { https://www.youtube.com/ }}{\text { user/elrubius0MG/ }}$ \\
\hline Willyrex & España & $9,002,668$ & $09 / 08 / 2009$ & 3756 & $\begin{array}{c}\underline{\text { https://www.youtube.com/ }} \\
\text { user/Willyrex/ }\end{array}$ \\
\hline VEGETTA777 & España & $20,413,438$ & $02 / 05 / 2008$ & 4207 & $\begin{array}{c}\text { https://www.youtube.com/ } \\
\text { user/vegetta777/ }\end{array}$ \\
\hline DjMaRiiO & España & $3,364,853$ & $10 / 12 / 2009$ & 1765 & $\begin{array}{c}\text { https://www.youtube.com/ } \\
\text { user/DjMaRiio/ }\end{array}$ \\
\hline
\end{tabular}

Tabla 1:Información de los perfiles de YouTubers.

En cuanto al análisis de los perfiles de los YouTubers considerados influencers, se ha observado que los destinatarios más habituales de estos canales son en su mayoría jóvenes, sin olvidar la importancia que tiene para cada YouTuber la presencia y representación de empresas en sus canales (véase Figura III).

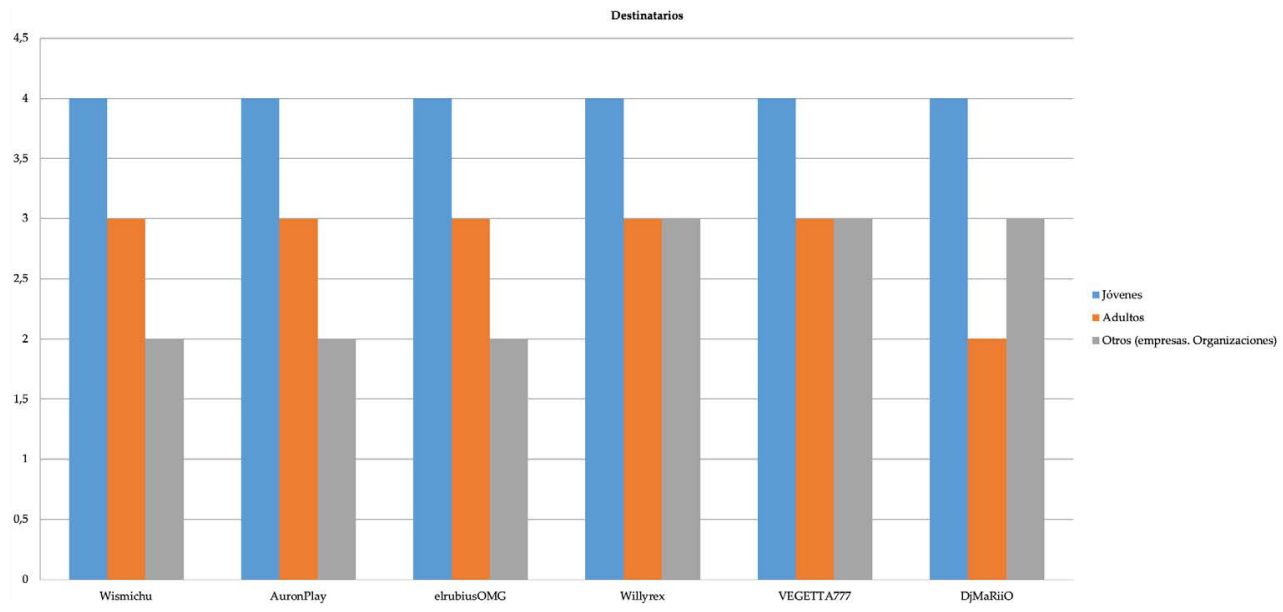

Figura 3: Destinatarios de los YouTubers. Fuente: Elaboración propia. 
Como se puede observar en la Figura IV, la mayoría de estos YouTubers son seguidos por hombres, principalmente porque las temáticas sobre las que tratan suelen ser videojuegos de deportes como el fútbol o de violencia; destacando Vegetta777, Willyrex y DjMario en los que aparecen contenidos más generales relacionados con bromas.

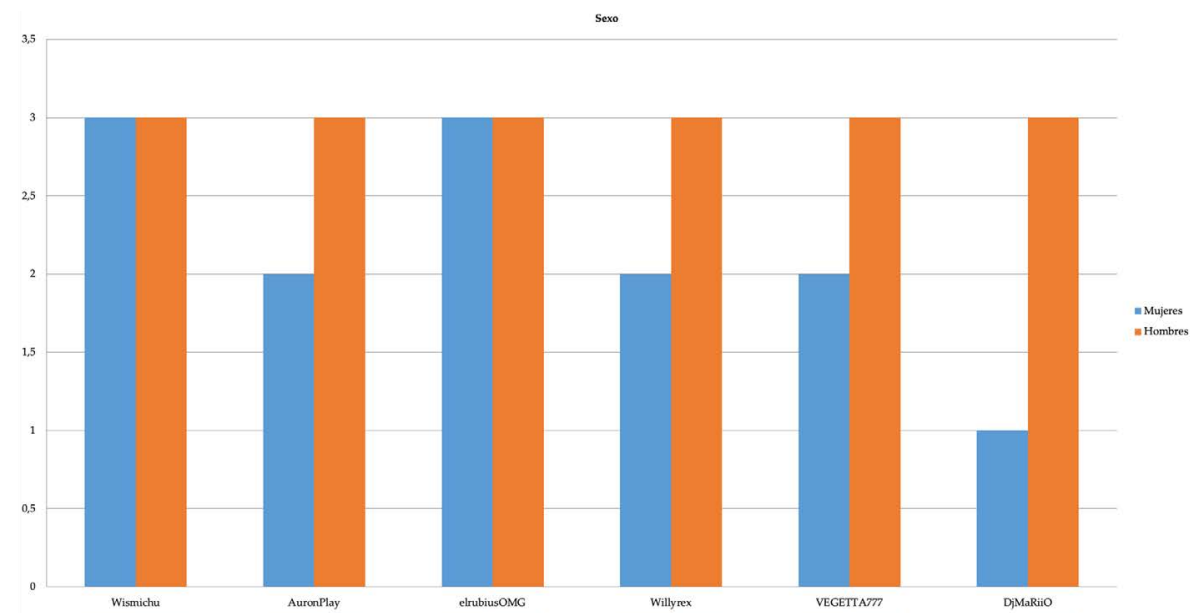

Figura 4: Sexo al que suele estar dirigido cada canal. Fuente: Elaboración propia.

En cuanto a las dimensiones de análisis, se destacan las siguientes: fotografía y descripción de cada perfil YouTuber, tipología de vídeos compartidos, opciones de interface con sus seguidores (comentarios y suscriptores) y contenidos y valores transmitidos en el canal.

\section{Fotografía y descripción de cada perfil YouTuber}

En relación con las fotografías que aparecen en los perfiles de los YouTubers (influencers) se destaca que la mayoría recurren a objetos mientras que sólamente AuronPlay muestra su rostro y Wismichu y Vegetta777 ofrecen caricaturas de sí mismos, el primero de su rostro únicamente y el segundo un plano medio emulando al protagonista de algún videojuego fantástico, tal y como él describe en su contenido del perfil (véase Figura V). En el caso de elrubuisOMG muestra el perfil de la 
YouTube e influencers en la infancia. Análisis de contenidos y propuestas educativas | 283

cabeza de gato troleado, aspecto que suele definirle en sus vídeos; Willyrex muestra solamente la letra W de su inicial y DjMariio tiene representado en su fotografía del perfil su nombre imitando el logo de su equipo de fútbol favorito y aludiendo a la temática que trata en su canal: fútbol y videojuegos del deporte.
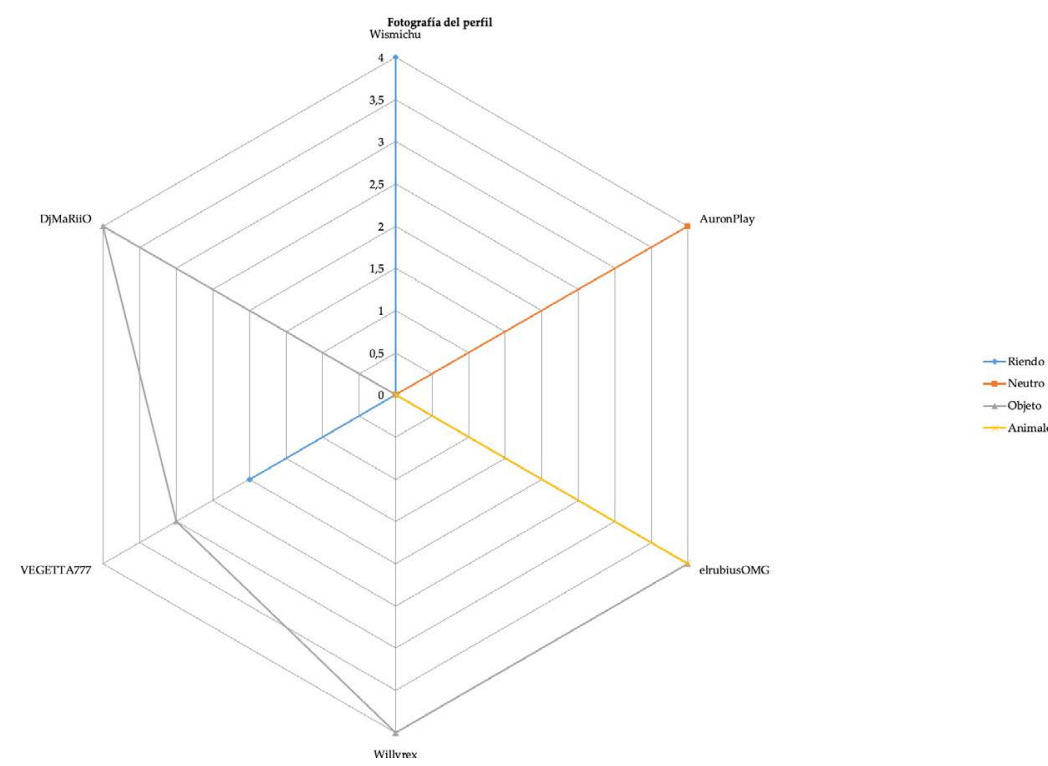

Figura 5: Contenido visual que aparece en el perfil de cada YouTuber.

Fuente: Elaboración propia.

\section{Tipología de vídeos compartidos}

Tomando como referencia la Figura VI, se ha analizado si en este caso cada YouTuber aparece solo o en compañía de otras personas. Se puede observar que Wismichu, AuronPlay, elrubiusOMG o DjMariio son quienes aparecen en ocasiones con amigos u otros Youtubers. En el caso de Willyrex o Vegetta777, al centrarse especialmente en los videojuegos, normalmente aparecen solos, aunque se pueden escuchar en los vídeos voces de personas con las que juegan conectados a través de la red. 


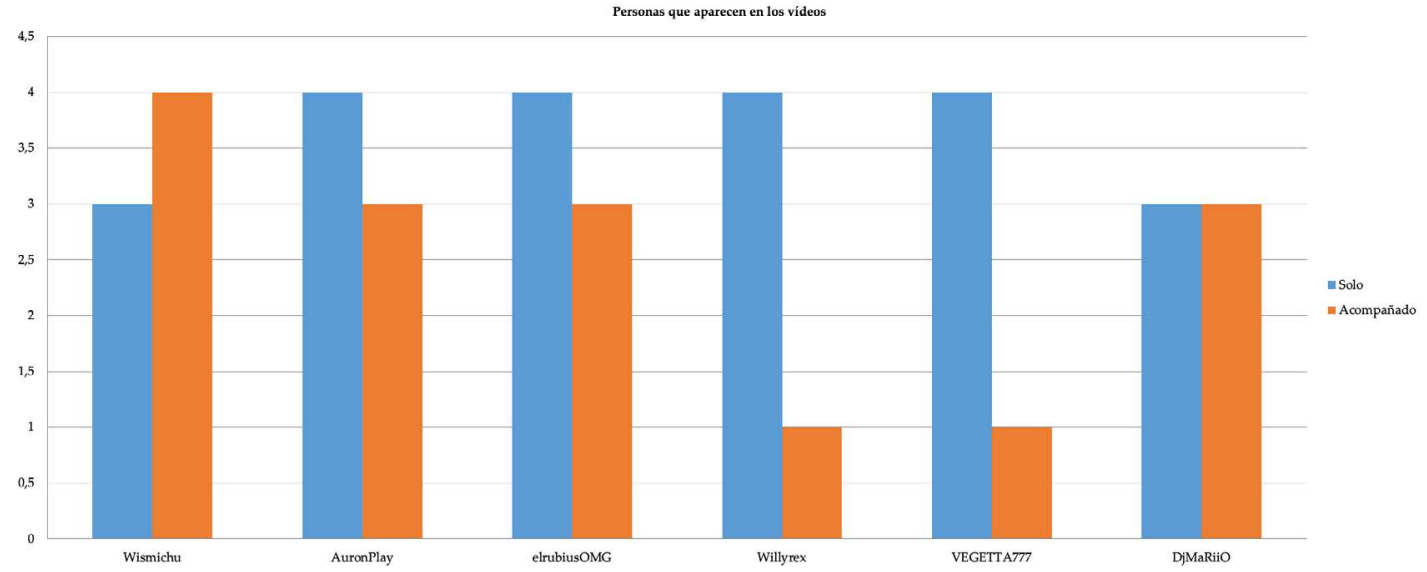

Figura 6: Personas que aparecen en los vídeos de cada YouTuber.

Fuente: Elaboración propia.

En cuanto al análisis de los vídeos en la Tabla II, se puede observar la estructura habitual de cada uno de ellos en función del YouTuber descrito, su sello de identidad y algunas expresiones que suelen emplear, destacando que en general suelen presentar inicios y finales en los vídeos con expresiones verbales o escritas comunes.

\begin{tabular}{|c|l|l|l|}
\hline YouTubers & $\begin{array}{c}\text { Estructura } \\
\text { inicial y final de } \\
\text { los videos }\end{array}$ & Sello de identidad & \multicolumn{1}{|c|}{$\begin{array}{c}\text { Expresiones } \\
\text { recurrentes }\end{array}$} \\
\hline Wismichu & $\begin{array}{l}\text { No inicia siempre sus } \\
\text { vídeos igual, algunos } \\
\text { son directamente } \\
\text { él hablando y otros } \\
\text { introduce música y } \\
\text { texto previo. }\end{array}$ & $\begin{array}{l}\text { Suele trolear a empresas } \\
\text { o famosos, generando } \\
\text { polémica y gastando } \\
\text { bromas. }\end{array}$ & $\begin{array}{l}\text { No sé si os acordáis } \\
\text { de un vídeo que subí } \\
\text { hace... }\end{array}$ \\
\hline AuronPlay & $\begin{array}{l}\text { Suele iniciar sus ví- } \\
\text { deos preguntando a } \\
\text { los suscriptores si es- } \\
\text { tán bien, y, además, } \\
\text { suele emplear el } \\
\text { término "chavales". } \\
\text { Finaliza despidiéndo- } \\
\text { se hasta el siguiente } \\
\text { vídeo. }\end{array}$ & $\begin{array}{l}\text { Bromas telefónicas, } \\
\text { comentar vídeos, simular } \\
\text { doblajes y aconsejar. }\end{array}$ & $\begin{array}{l}\text { iQué pasa chavales! } \\
\text { ¿Todo bien, todo } \\
\text { correcto? }\end{array}$ \\
\hline
\end{tabular}


MONOGRÁFICO

\begin{tabular}{|c|c|c|c|}
\hline YouTubers & $\begin{array}{c}\text { Estructura } \\
\text { inicial y final de } \\
\text { los videos }\end{array}$ & Sello de identidad & $\begin{array}{l}\text { Expresiones } \\
\text { recurrentes }\end{array}$ \\
\hline elrubius0MG & $\begin{array}{l}\text { Suele iniciar sus } \\
\text { vídeos saludando a } \\
\text { sus suscriptores con } \\
\text { la misma expresión } \\
\text { y suele finalizar so- } \\
\text { licitando likes a sus } \\
\text { seguidores en forma } \\
\text { de texto escrito. } \\
\end{array}$ & $\begin{array}{l}\text { Simula sustos y miedos, } \\
\text { representa situaciones } \\
\text { cotidianas de su vida } \\
\text { exageradas, y es especia- } \\
\text { lista en random, }\end{array}$ & $\begin{array}{l}\text { Muy buenas, criaturi- } \\
\text { tas del terror }\end{array}$ \\
\hline Willyrex & $\begin{array}{l}\text { Inicia sus vídeos } \\
\text { saludando a sus } \\
\text { suscriptores con la } \\
\text { misma expresión } \\
\text { exceptuando si son } \\
\text { vídeos de videojue- } \\
\text { gos que continúan } \\
\text { de otros días y suele } \\
\text { finalizar con un } \\
\text { adiós y saludando } \\
\text { con la mano. }\end{array}$ & $\begin{array}{l}\text { La mayoría de sus vídeos } \\
\text { se centran en videojue- } \\
\text { gos y en ofrecer solucio- } \\
\text { nes o buenas partidas. } \\
\text { También tiene los blogs } \\
\text { sobre experiencias perso- } \\
\text { nales y también realiza } \\
\text { directos. }\end{array}$ & $\begin{array}{l}\text { Muy buenas a todos, } \\
\text { aquí Willyrex comen- } \\
\text { tando... }\end{array}$ \\
\hline VEGETTA777 & $\begin{array}{l}\text { Saluda siempre en } \\
\text { sus vídeos con la } \\
\text { misma expresión y } \\
\text { finaliza con un besa- } \\
\text { zo y chao chao }\end{array}$ & $\begin{array}{l}\text { Ofrece vídeos sobre vi- } \\
\text { deojuegos principalmen- } \\
\text { te de Minecraft. Suele } \\
\text { usar un lenguaje correcto } \\
\text { en sus vídeos. }\end{array}$ & $\begin{array}{l}\text { Hey, muy buenas a } \\
\text { todos, guapisimos... }\end{array}$ \\
\hline DjMaRiiO & $\begin{array}{l}\text { Siempre suele sa- } \\
\text { ludar y despedirse } \\
\text { en sus vídeos, pero } \\
\text { no sigue una unas } \\
\text { expresiones rutina- } \\
\text { rias, a veces suele } \\
\text { iniciar los vídeos con } \\
\text { música. }\end{array}$ & $\begin{array}{l}\text { Sus vídeos siempre están } \\
\text { relacionados con el fút- } \\
\text { bol, su pasión. }\end{array}$ & $\begin{array}{l}\text { Tenemos un equipo } \\
\text { impresionante }\end{array}$ \\
\hline
\end{tabular}

Tabla 2: Información de los vídeos de YouTubers.

\section{Opciones de interfaz con sus seguidores}

En relación con el análisis de los comentarios y sus suscriptores, es de destacar que los seis YouTubers suelen dejar visibles los comentarios que hacen sus followers y además, suelen seguir algunas conversaciones respondiendo a dudas o consultas 
286 | Paula Renés Arellano, Vicent Gozálvez Pérez e Inmaculada Berlanga Fernández MONOGRÁFICO

de estos. Además, al finalizar sus vídeos suelen invitar no solo a darle a Me gusta en ese vídeo sino también a seguir viéndole en el próximo vídeo.

\section{Contenidos y valores transmitidos en el canal}

Sobre el contenido que aparece en los vídeos y los valores que se transmiten, cabe destacar que en general la mayoría no cuida el lenguaje que utiliza, solamente Vegetta777 suele evitar emplear palabras malsonantes y si lo hace suele pedir disculpas (véase Figura VII).

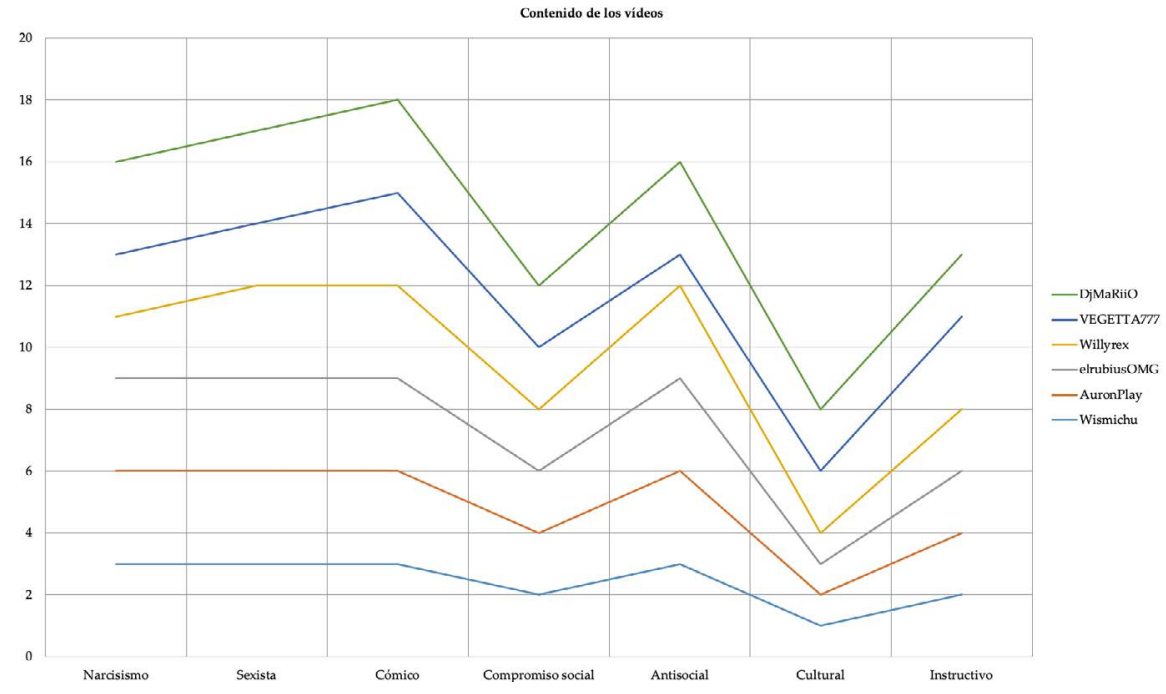

Figura 7: Tipo de contenido que suele aparecer en los vídeos de cada YouTuber. Fuente: Elaboración propia.

En el caso de Wismichu, este suele realizar vídeos provocadores, sin cuidar el lenguaje ni las formas, y suele utilizar lenguaje sexista; y aunque podemos clasificar muchas de sus bromas como "pesadas" y poco educativas, no es menos cierto que está muy implicado en la defensa de los animales, identificándose como vegetariano y antitaurino. Con AuronPlay también ocurre parecido en tanto que no cuida el lenguaje que emplea en los vídeos, aunque las bromas que realiza no son tan exageradas. 
Tomando como referencia a elrubiusOMG, este suele representar y dramatizar las jugadas que realiza en los videojuegos, pero sin controlar el lenguaje, con palabrotas en mucha ocasiones y comentarios sexistas y no pertinentes de los personajes o jugadas realizadas.

Willyrex suele trabajar en ocasiones en colaboración con Vegetta777, centrándose más en videojuegos online y en jugadas, siendo este último quien suele ofrecer sus partidos a Minecraft y cuidando el lenguaje que emplea, centrándose en las partidas que hace, graba y luego comparte.

Finalmente, DjMaRiiO está centrado en simular partidos de fútbol, retransmitiéndolos o proyectándolos a sus followers, aunque sin cuidar el lenguaje que utiliza.

\section{Discusión y conclusiones}

Los Youtubers que se han descrito y analizado anteriormente se han convertido en fenómenos de masas, y si bien algunos tienen más fama internacional y suscriptores en las redes, lo cierto es que los niños y jóvenes españoles consumen sus contenidos publicados en los canales. En relación con el objetivo sobre el análisis del contenido que consumen los niños de los Youtubers más seguidos, se observa que las jugadas que realizan, graban y emiten en los canales en torno a diversos videojuegos con humor, a veces controladas y en otras ocasiones sin cuidar el lenguaje ni el contenido, obliga a pensar sobre cuál es el valor educativo de los contenidos que ven y consumen los niños en YouTube a través del móvil, partiendo de que la propia plataforma promueve que la persona sea activa (Ritzer, Dean, \& Jurgeson, 2012), aspecto que se puede observar en el apartado de Tipología de vídeos e Información de los vídeos. Las parodias que hacen de famosos, las bromas telefónicas y otros contenidos similares provocan que los niños y jóvenes se rían y les sigan, lo cual de una u otra manera está influyendo en su desarrollo sociomoral y en la construcción de su identidad moral (Ameneiros y Ricoy, 2015; Sánchez-Teruel, \& Robles-Bello, 2016; Palomino, Arroyave y Londoño, 2018). Tal y como se ha reflejado en los resultados, la mayoría de Youtubers invitan al final de sus vídeos a seguirles, o dar Me gusta, independientemente de si el contenido es adecuado o no. La cuestión es qué valores están transmitiendo estos influencers y 
cuáles están aprendiendo sus seguidores, niños y niñas de nuestra sociedad, porque los YouTubers seleccionados transmiten en sus vídeos contenidos que no están a la altura ética de una ciudadanía democrática (Gozálvez, 2013), dado su carácter tanto sexista como antisocial (trato vejatorio al otro, uso instrumental de la persona como objeto de burlas, uso del "troleo" como forma de manipular el diálogo...), lo que hace necesario pensar y reflexionar sobre el tipo de estrategias educativas recomendables tanto en la escuela como en la familia ante la irrupción de estos contenidos en redes sociales como YouTube.

Realmente, "las redes sociales no deben entenderse como simples herramientas tecnológicas para el intercambio de mensajes" (García, del Hoyo \& Fernández, 2014:36), detrás de ellas hay significados y significantes, mensajes y contenidos que incorporan valoraciones sobre la realidad y que influyen de manera directa 0 indirecta en los jóvenes seguidores, un claro ejemplo se inicia con la fotografía y descripción de cada perfil Youtuber. Algunas investigaciones recientes sobre los contenidos de vídeos publicados en YouTube indican el uso problemático del lenguaje y las técnicas audiovisuales en los mismos, incidiendo en la necesidad de promover códigos adecuados entre los jóvenes que emiten vídeos en estas redes para evitar riesgos, especialmente, en relación con el uso adecuado de su identidad digital (Frutos, Sánchez y Vázquez, 2014; Montes-Vozmediano, García-Jiménez \& Menor-Sendra, 2017).

Siguiendo las recomendaciones planteadas en estudios como los citados, y en otros como el de Escobar-Román (2011), que incide en los efectos que pueden ocasionar el uso de redes sociales, o de Callejo \& Gutiérrez (2014) o Morgan \& Kristjánsson (2017), que proponen el diálogo como herramienta de interés para abordar esta temática, formulamos algunas propuestas con el fin de promover el uso adecuado de este tipo de plataformas entre los niños y jóvenes, tanto en ambientes escolares como en la familia, siendo este último agente educativo un pilar sustancial en el mundo de las nuevas redes (Rumayor, 2016):

- Favorecer una mirada crítica y reflexiva sobre los discursos de los Youtubers, para (1) promover comportamientos igualitarios y no estereotipados, (2) evitar la ciberviolencia machista que puede percibirse en YouTube, y (3) buscar 
que la plataforma se convierta en un auténtico banco de recursos audiovisuales que puedan emplearse de forma (co)educativa (Linares-Bahillo, AristeguiFradua \& Beloki-Marañón, 2019).

- Elaboración de recursos didácticos que permitan el análisis de este tipo de plataformas sociales en las escuelas. Tal y como afirman Tur-Viñes, NúñezGómez \& González-Río (2018) en sus recomendaciones sobre la necesidad de regular y gestionar adecuadamente los canales de YouTube, cabe educar especialmente a los menores con la finalidad de que sea un entorno seguro y responsable.

- Regular adecuadamente por parte de las instituciones políticas y sociales el tipo de perfiles y canales a los que tienen acceso los niños y jóvenes de forma habitual. Aspectos que de forma progresiva parece que se están percibiendo, tal y como refleja el nuevo protocolo que este año Autocontrol, la Asociación Española de Anunciantes (AEA) y la Secretaría de Estado de Avance Digital están preparando a través de un código específico sobre la utilización de Influencers en la publicidad, respaldado por decisiones previas tomadas en países pioneros como Reino Unido con Advertising Standards Authority (ASA), que identificó en 2018 la necesidad de regular adecuadamente las publicaciones patrocinadas en las redes sociales.

- Empoderar a los profesores y a las familias para ser competentes en la educación digital y mediática de sus hijos y alumnos, porque como afirman Pereira, Fillol \& Moura (2019: 49): “Los profesores tienen un papel importante en el empoderamiento de los alumnos ante los problemas de la era de la información digital, pero, para ello, deben tener formación y la alfabetización mediática".

- Promover una verdadera educación en medios que conecte el centro escolar con las necesidades y demandas sociales (Arnaiz \& De Haro, 2004), en el que se revalorice más el qué seguir, que a quién seguir (Izquierdo-Iranzo y Gallardo-Echenique, 2020).

- Desarrollar acciones formativas orientadas a promover modelos educativos familiares que permitan a las familias fortalecer su autoridad ante las nuevas redes sociales, para lo que se requieren procesos de formación en la línea del 
MONOGRÁFICO

uso adecuado y éticos de los nuevos marcos virtuales de interacción social y personal. En esta línea se destaca la aportación de Labrador, Requenses \& Helguera (2015) a través de una guía preventiva sobre usos adecuados de Internet.

- Impulsar de modo general la educación o alfabetización mediática (Unesco, 2011) con el fin de aprovechar las potencialidades de redes sociales en cuanto a la formación sociomoral en la infancia y juventud, tal como sugieren Koh (2014) o Banaji (2013), y contemplando asimismo el tratamiento pedagógico de los riesgos o peligros vinculados a las redes.

Por tanto, se hace necesario seguir investigando sobre los efectos que pueden generar el seguimiento de determinados YouTubers entre niños y niñas menores de edad ya que, en palabras de Garduño \& Sting (2017: 231), la nueva figura de las personas que consumen medios "es la nueva unidad cultural que se compromete con la posibilidad de mantener su propio desarrollo a costa de "lo" demás y de "los" demás sin necesidad de referencia trascendental". Y se estima aún más necesaria la creación de medidas preventivas para evitar problemas en el desarrollo de la construcción moral de los niños y niñas. Es esta una línea defendida por los autores mencionados y a su vez por autores como Rodríguez \& Magdalena (2016), quienes consideran necesario e imprescindible dar respuesta a necesidades y carencias actuales de los jóvenes en torno al uso adecuado de la sociales virtuales.

\section{Agradecimientos}

Este trabajo se enmarca en 'Alfamed' (Red Euroamericana de Investigadores), con el apoyo del Proyecto I+D “ YOUTUBERS E INSTAGRAMMERS: LA COMPETENCIA MEDIATICA EN LOS PROSUMIDORES EMERGENTES" (RTI2018-093303-B-I00), financiado por la Agencia Estatal de Investigación del Ministerio de Ciencia, Innovación y Universidades de España y el Fondo Europeo de Desarrollo Regional (FEDER).

\section{Referencias}

Alloway, T.P., Horton, J., Alloway, R.G. \& Dawson, C. (2013). Social networking sites and cognitive abilities: Do they make you smarter? Computer and Education, 63, 10-16. doi: https://doi.org/10.1016/j.compedu.2012.10.030 
Ameneiros, A. \& Ricoy, M. C. (2015). Los videojuegos en la adolescencia: prácticas y polémicas asociadas. Revista de estudios e investigación en psicología y educación, 13, 115-119. doi: https://doi.org/10.17979/reipe.2015.0.13.451

Arnaiz, P. \& De Haro, R. (2004). Ciudadanía e interculturalidad: claves para la educación del siglo XXI. Educatio, 22, 19-37.

Arthur, J., Kristjánsson, K., Harrison, T., Sanderse, W. \& Wright, D. (2017). Teaching Character and Virtue in Schools. New York: Routledge.

Banaji, S. (2013). Everyday racism and «My Tram Experience». Emotion, civic performance and learning on YouTube. Comunicar, 40, 69-78. doi: https://doi. org/10.3916/C40-2013-02-07.

Benhabib, S. (2005). Los derechos de los otros: Extranjeros, residentes y ciudadanos. Barcelona: Gedisa.

Blackwell, C.K., Lauricella, A.R., Conway, A. \& Wartella, E. (2014). Children and the Internet: Developmental Implications of Web Site Preferences Among 8- to 12-Year-Old Children. Journal of Broadcasting \& Electronic Media, 58, 1-20. doi: https://doi.org/10.1080/08838151.2013.875022

Briggs J. (2017). Young people and participation in Europe. London: Palgrave Macmillan. doi: https://doi.org/10.1057/978-1-137-31385-0

Callejo, J. \& Gutiérrez, J. (2014). La comunicación de los jóvenes adolescentes en las redes sociales. Revista interdisciplinar de estudios de comunicación y ciencias sociales, 19, 11-29. Recuperado de http://hdl.handle.net/10637/6618 Conill, J. (2006). Ética hermeneútica. Madrid: Tecnos.

Cortina, A. (2007). Ética de la razón cordial. Educar en la ciudadanía del siglo XXI. Madrid: Nobel.

Cortina, A. (2010). Justicia cordial. Madrid: Trotta.

De-Frutos, B. \& Marcos, M. (2017). Disociación entre las experiencias negativas y la percepción de riesgo de las redes sociales en adolescentes. El Profesional de la Información, 26(1), 88-96. doi: https://doi.org/10.3145/epi.2017.ene.09

Escobar, M. \& Román, H. (2011). La presentación del yo en el ciberespacio: un análisis de las autodefiniciones personales en blogs y redes sociales. Revista de Psicología Social, 26(2), 207-222. doi: https://doi.org/10.1174/021347411795448947

Frutos Torres, B., Sánchez Valle, M., \& Vázquez Barrio, T. (2014): Perfiles de adolescentes online y su comportamiento en el medio interactivo. Icono 14, 12(1), 374-397. doi: https://doi.org/10.7195/ri14.v12i1.208 
García, M.C., del Hoyo, M., \& Fernández, C. (2014). Jóvenes comprometidos en la Red: el papel de las redes sociales en la participación social activa. Comunicar, 43, 35-43. doi: https://doi.org/10.3916/C43-2014-03

Garduño, G., \& Sting, R. (2017). Desarrollo, mito y discurso. La configuración del mundo de consumo a través del lenguaje. Revista de Comunicación 16(2), 214233. doi: https://doi.org/10.26441/RC16.2-2017-A10

Gozálvez, V. (2013). Ciudadanía mediática. Una mirada educativa. Madrid: Dykinson. Gozálvez, V., \& Jover, G. (2016). Articulación de la justicia y el cuidado en la educación moral: Del universalismo sustitutivo a una ética situada de los derechos humanos. Educación XXI, 19(1), 311-330. doi: https://doi. org/10.5944/educXX1.14221

Harrison, T. (2014). The influence of the Internet on the character virtues of 11-14 year olds. Birmingham: University of Birmingham.

Harrison, T. (2016). Virtuous reality: moral theory and research into cyber-bullying. Ethics \& Information Technology, 17(4), 275-283. D0I 10.1007/s10676-0159382-9

Holloway, D., Green, L. \& Livingstone, S. (2013). Zero to eight: young children and their internet use. EU Kids Online. LSE London.

Informe Ditrendia (2018). Informe Mobile en España y en el mundo 2018. Recuperado de https://ditrendia.es/informe-ditrendia-mobile-en-espana-yen-el-mundo-2018

ITU (2019). Measuring digital development. Facts and figures, 2019. Recuperado de https://itu.foleon.com/itu/measuring-digital-development/home/

Izquierdo-Iranzo, P. \& Gallardo-Echenique, E.E. (2020). Studygrammers: Learning influencers. [Estudigramers: Influencers del aprendizaje]. Comunicar, 62, 115125. https://doi.org/10.3916/C62-2020-10

Koh, C. (2014). Exploring the use of Web 2.0 technology to promote moral and psychosocial development: Can YouTube work? British Journal of Educational Technology, 45(4), 619-635. doi: https://doi.org/10.1111/bjet.12071

Kohlberg, L. (1984). Psicología del desarrollo moral. Bilbao: Desclée de Brouwer. Kurtines W.M. \& Gewirtz J.L. (Eds.) (1991). Handbook of moral behavior and development. Hillsdale, NJ: Erlbaum. 
YouTube e influencers en la infancia. Análisis de contenidos y propuestas educativas | 293 MONOGRÁFICO

Labrador, F., Requenses, A., \& Helguera, M. (2015). Guía para padres y educadores sobre el uso seguro de Internet, móviles y videojuegos. Madrid: Fundación Gaudium. Recuperado de https://goo.gl/wtQCvB

Linares-Bahillo, E., Aristegui-Fradua, I. \& Beloki-Marañón, U. (2019). YouTube, una plataforma para la (in)formación, relación, comunicación, diversión, y gestión de identidades (de género) en la natividad digital. Revista Mediterránea de Comunicación: Mediterranean Journal of Communication, 10(1), 55-70. doi: https://doi.org/10.14198/MEDCOM2019.10.1.18

Loevinger, J. (1987). Paradigms of Personality. Nueva York: Freeman.

López Noguero, F. (2002). El análisis de contenido como método de investigación. XXI. Revista de Educación, 4, 169-179.

Montes-Vozmediano, M.; García-Jiménez; A., \& Menor-Sendra, J. (2017). Los vídeos de los adolescentes en YouTube: Características y vulnerabilidades digitales. Comunicar, 54, 61-69. doi: https://doi.org/10.3916/C54-2018-06

Morgan, B. \& Kristjánsson, K. (2017). Parents and Social Media. Adolescents' perceptions of parental responses to morally salient social media scenarios. Birmingham: University of Birmingham and The Jubilee Center for Character and Virtues

Nucci, L. (2001). Education in the moral domain. Cambridge, M.A.: Cambridge University Press.

Nussbaum, M. (2012). Crear capacidades. Propuesta para el desarrollo humano. Barcelona: Paidós.

Palomino, M., Arroyave, y Londoño, 0. (2018). Actitudes prosociales en una muestra de niños de 7 a 10 años de una institución educativa oficial. Revista Katharsis, 25, 37-54. Recuperado de http://revistas.iue.edu.co/index.php/katharsis

Pereira, S., Fillol, J. \& Moura, P. (2019). El aprendizaje de los jóvenes con medios digitales fuera de la escuela: De lo informal a lo formal. Comunicar, 58, 41-50. doi: https://doi.org/10.3916/C58-2019-04

Pérez-Escoda, A. \& Contreras, P. (2018). Smartphone y redes sociales para el desarrollo de competencias mediáticas y digitales en niños y adolescentes: Musical.ly. Aula Abierta, 47(3), 281-290. doi: https://doi.org/10.17811/ rifie.47.3.2018.281-290 
294 | Paula Renés Arellano, Vicent Gozálvez Pérez e Inmaculada Berlanga Fernández MONOGRÁFICO

Ritzer, G., Dean, P., \& Jurgenson, N. (2012). The Coming of Age of the Prosumer. American Behavioral Scientist, 56(4), 379-398. doi: https://doi. org/10.1177/0002764211429368

Rodríguez García, L., \& Magdalena Benedito, J.R. (2016): Perspectiva de los jóvenes sobre seguridad y privacidad en las redes sociales, Icono 14, volumen (14), pp. 24-49. doi: http://dx.doi.org/10.7195/ri14.v14i1.885

Rumayor, M. (2016). El problema de la autoridad en la familia frente al reto de las redes sociales. Teoría de la Educación. Revista Interuniversitaria [Internet]. 28(2): 75-92. doi: http://dx.doi.org/10.14201/teoredu2827592

Sánchez-Teruel, D. \& Robles-Bello, M. A. (2016). Riesgos y potencialidades de la era digital para la infancia y la adolescencia. Revista Educación y Humanismo, 18(31), 186-204. doi: http://dx.doi.org/10.17081/eduhum.18.31.1374

Santana-Vega, L., Gómez-Muñoz, A. \& Feliciano-García, L. (2019). Uso problemático del móvil, fobia a sentirse excluido y comunicación familiar de los adolescentes. Comunicar, 59, 39-47. doi: https://doi.org/10.3916/C59-2019-04

Sabater, C. (2014). La vida privada en la sociedad digital. La exposición pública de los jóvenes en Internet. Aposta, 61, 1-32. Recuperado de https://goo. gl/OHRnyI

Tur-Viñes, V., Núñez-Gómez, P. \& González-Río, M. J. (2018). Menores influyentes en YouTube. Un espacio para la responsabilidad. Revista Latina de Comunicación Social, 73, 1211-1230. doi: https://doi.org/10.4185/RLCS-2018-1303

Turiel, E. (1998). The development of morality. En N. Eisenberg (Ed.). Handbook of child psychology: Vol 3. Social, emotional, and personality development (pp. 863-932). NY: Wiley.

UNESCO (2011). Alfabetización mediática e informacional. Curriculum para profesores. París: UNESCO.

Vizcaíno-Verdú, A., Contreras, P. \& Guzmán-Franco, M. (2019). Lectura y aprendizaje informal en YouTube: El booktuber. Comunicar, 59, 95-104. doi: https://doi. org/10.3916/C59-2019-09

Yañez-Canal, J., Billmann, E., Mójica, Y.A. \& Perdomo, A. (2013). Sociomoral reasoning in Children with respect to exclusion and inclusion relationships, Psychology Research, 3 (12), 719-736. doi: https://doi.org/10.17265/21595542/2013.12.004 
YouTube e influencers en la infancia. Análisis de contenidos y propuestas educativas | 295 MONOGRÁFICO

Zahn, C., Schaeffeler, N., Giel, K.E., Wessel, D. Thiel, A., Zipfel, S., \& Hesse, F. W. (2014). Video clips for YouTube: Collaborative video creation as an educational concept for knowledge acquisition and attitude change related to obesity stigmatization. Education and Information Technologies, 19, 603-621. doi: https://doi.org/10.1007/s10639-013-9277-5

Zayas, B., Gozálvez, V. \& Gracia, J. (2019). La dimensión ética y cívica del Aprendizaje-Servicio: Un modelo para su institucionalización en la Educación Superior. Revista Complutense de Educación, 30 (1), 1-15.

\section{(c)}

Este obra está bajo una licencia de Creative Commons Reconocimiento 4.0 Internacional. 